\title{
Les cellules souches mésenchymateuses
}

\section{Actualités thérapeutiques}

> Les cellules souches/stromales mésenchymateuses sont une population cellulaire capable d'agir sur la réparation et la régénération tissulaires par différents mécanismes allant du remplacement cellulaire à la modulation des réponses immunitaires et inflammatoires. Du fait de leurs différentes potentialités, les CSM ont été utilisées dès 1995 d'abord dans un contexte hématologique pour aider au soutien de l'hématopoïèse, puis pour traiter la réaction du greffon contre l'hôte. Leur potentiel de différenciation explique également leur utilisation dans un but de réparation osseuse. Depuis plus de 15 ans les champs d'application potentiels étudiés dans des essais cliniques se sont étendus à des indications aussi variées que les lésions cutanées au cours des brûlures ou les accidents vasculaires cérébraux. Le but des textes regroupés dans cet article n'est pas de donner une vision exhaustive de l'ensemble des indications potentielles en cours d'évaluation, mais de montrer ce que peuvent être des applications combinant les différents moyens d'action des CSM dans quatre domaines particuliers: les pathologies dysimmunitaires, la réparation osseuse, la régénération vasculaire et l'ophtalmologie. <

Nous faisons le point dans cette série d'articles sur les multiples applications thérapeutiques des cellules souches mésenchymateuses (CSM) : maladies autoimmunes dont le diabète, le lupus, la sclérose en plaques et la maladie de Crohn; affections cardiovasculaires; régénération des tissus ostéoarticulaires et prévention de la GVHD (graft versus host disease). Plus de 140 essais cliniques utilisant ces cellules sont actuellement répertoriées sur le site clinicaltrials.gov dont 22 concernent les maladies auto-immunes. Beaucoup de ces essais sont en cours, les résultats ne sont pas encore tous publiés mais leur

nombre témoigne d'une activité croissante dans ces indications. Les CSM sont des cellules progénitrices adultes essentiellement isolées de la moelle osseuse et du tissu adipeux mais présentes dans la plupart des tissus adultes, y compris les muscles, le tissu synovial, le tissu placentaire et, récemment, les dents. Les indications thérapeutiques des CSM reposent sur deux concepts. Le premier concept utilise les CSM dans l'ingénierie tissulaire, par création de tissus osseux, cartilagineux ou musculaire en combinaison avec des biomatériaux adaptés, comme l'exposent Frédéric Deschaseaux (voir plus loin) et Claire Vinatier et al. $(\rightarrow$ ) mais peut être aussi cardiovasculaire (V. PlanatBenard, voir plus loin).

$\rightarrow$ Voir l'article de C. Vinatier et al., page 289 de ce numéro Un concept thérapeutique plus récent repose sur leurs propriétés paracrines, liées à la sécrétion de nombreux facteurs de croissance dont HGF (hepatocyte growth factor ), IGF (insulin growth factor), TGF- $\beta$ (transforming growth factor beta) mais également des molécules anti-inflammatoires incluant des cytokines, IL-lra (antagoniste du récepteur de l'interleukine 1), IDO (indoleamine 2,3-dioxygénase) et

$(\rightarrow)$ Voir l'article de C. Ménard et K. Tarte, page 269 de ce numéro HLA-G5 $(\rightarrow)$.

L'effet immunomodulateur des CSM sur les cellules dendritiques et/ ou sur les lymphocytes $T$ et $B$ suggère effectivement leur utilisation potentielle dans les maladies dysimmunitaires, mais aussi leur intérêt dans les maladies cardiovasculaires (V. Planat-Benard, voir plus loin) ou ophtalmologiques ( $\varepsilon$. Gabison, voir plus loin). 


\section{Les cellules souches mésenchymateuses \\ dans les maladies dysimmunitaires \\ Christian Jorgensen}

Les capacités immunosuppressives des CSM décrites en détail par C. Ménard et K. Tarte $(\rightarrow)$ ont été évaluées dans des modèles expérimentaux de maladies auto-immunes dont l'experimental autoimmune encephalopathy $(\varepsilon A \varepsilon)$, le diabète,

$\rightarrow$ Page 269 de ce numéro. l'arthrite ou le lupus. Dans un modèle de diabète auto-immun, l'injection de CSM a induit une diminution de l'épaississement mésangial et de l'infiltration macrophagique du pancréas.

\section{Maladie lupique}

Dans la maladie lupique, caractérisée par l'hyperactivité lymphocytaire B, la production d'auto-anticorps sous forme d'anticorps antinucléaires, anti-ADN et anti-SM ${ }^{1}$, quatre études sont en cours. Une première étude chinoise a été publiée portant sur 82 patients traités avec des doses de 1 à 10 millions de cellules par kg de poids [1]. II s'agit de cellules mésenchymateuses d'origine allogénique, issues de la moelle osseuse et amplifiées en culture. C'est une étude ouverte non contrôlée qui confirme la bonne tolérance de l'injection systémique de cellules mésenchymateuses mais ne permet pas d'affirmer un bénéfice clinique. Vingt-deux pour cent des patients sont répondeurs sur les critères BILAG (British Isles lupus assessment group) qui est utilisé pour évaluer cliniquement ces patients. Enfin, deux cas de lupus réfractaire traités par CSM autologues ont été publiés dans une autre étude [2]. Ces patients ont bénéficié d'injection de cellules mésenchymateuses d'origine médullaire autologues mais là encore sans bénéfice thérapeutique. Une des patientes a développé une insuffisance rénale liée au lupus. La tolérance des injections était acceptable et les auteurs ont pu constater une augmentation modeste des lymphocytes $T$ régulateurs circulants.

\section{Sclérose en plaques}

La sclérose en plaques est caractérisée par un infiltrat lymphocytaire T dans le système nerveux et une démyélinisation responsable d'épisodes neurologiques déficitaires récurrents. Dans le modèle d'encéphalite expérimentale $(\varepsilon A \varepsilon)$, l'injection systémique de CSM allogéniques montre un bénéfice quand l'injection se fait avant l'induction de l'encéphalite, mais elle n'a pas d'effet curatif. On constate alors une régression de l'infiltrat lymphocytaire $T$ et une diminution des lésions neurologiques centrales qu'accompagne l'amélioration du score clinique [3]. Ce travail montre que les CSM exercent un effet immunosuppresseur indirect par effet systémique et non pas par une action locale. Les CSM sont en effet retrouvées dans l'espace sous arachnoïdien mais pas au sein du système nerveux proprement dit. En revanche, l'efficacité clinique est

${ }^{1} \mathrm{Sm}$ désigne l'antigène Smith, premier autoantigène décrit en 1966 au cours du lupus, un complexe de protéines nucléaires et d'ARN. associée à la diminution significative de l'infiltrat monocytaire intracérébral et à une réduction de la démyélinisation. Plusieurs essais sont en cours chez l'homme; ils incluent des patients ayant des scléroses en plaques sévères. Une étude de phase I-II a été conduite chez 34 patients, qui ont reçu des doses de 1 à 4 millions de cellules par kg de poids. Ces patients ont bénéficié d'une injection intrathécale de CSM, et le score EDSS (expanded disability status score) s'est amélioré, passant de 6,7 à 5,9 avec une amélioration des données de l'imagerie. Un second travail publié [4] met en évidence une amélioration partielle du score EDSS chez 7 patients sur 10 [5]. Des études contrôlées sont nécessaires pour démontrer un bénéfice par rapport au traitement standard qui inclut l'interféron.

\section{Maladie de Crohn}

La maladie de Crohn, maladie intestinale inflammatoire, se complique fréquemment d'arthrite, d'uvéite et de fistulisation. Ces complications locales sont source de gêne fonctionnelle majeure et de surinfection. Une étude de phase II, incluant 49 patients, prospective et randomisée, a montré l'intérêt d'un traitement local par des cellules mésenchymateuses issues du tissu adipeux dans les cas de fistule [6]: 25 patients ont été traités par fibrine seule et 24 patients par fibrine associée à 20 millions de CSM. Une majorité de ces derniers $(71 \%)$ a montré une cicatrisation contre $16 \%$ seulement dans le groupe contrôle. L'action bénéfique se manifeste également par la réduction de l'inflammation et le raccourcissement du délai de cicatrisation locale. D'autres études sont en cours dont des études contrôlées de phase III qui vont pouvoir comparer des doses de 10 à 90 millions de cellules en injection locale versus un bras placebo.

\section{Diabète de type 1}

Le diabète de type 1 est une maladie auto-immune caractérisée par un infiltrat lymphocytaire T des îlots pancréatiques aboutissant à une destruction des cellules $\beta$ sécrétrices d'insuline. Dans des modèles expérimentaux de diabète, les injections de cellules mésenchymateuses humaines ont amélioré l'état clinique et la survie des animaux, ont réduit l'infiltrat lymphocytaire T pancréatique et entraîné une anergie lymphocytaire T. C'est donc à la suite de ces travaux qu'ont été proposées des injections de cellules mésenchymateuses allogéniques dans le diabète de type 1 . OSIRIS ${ }^{2}$ est promoteur de cette étude qui compare chez 60 patients répartis en 2 bras, soit 8 millions de cellules par $\mathrm{kg}$,

\footnotetext{
${ }^{2}$ Osiris est une compagnie privée américaine qui la première a commercialisé des cellules mésenchymateuses pour des applications thérapeutiques, sous le nom de Prochymal ${ }^{\circledR}$ (administration intraveineuse) et Chondrogen ${ }^{\circledR}$ pour l'arthrite.
} 
soit placebo. Les patients seront suivis pendant un an sur les marqueurs du diabète et notamment le taux d'hémoglobine glyquée (Hbalc).

\section{Arthrite expérimentale}

Dans l'arthrite expérimentale induite par le collagène chez l'animal, des travaux montrent une amélioration de l'inflammation articulaire suggérant un bénéfice thérapeutique potentiel des CSM dans la polyarthrite rhumatoïde. Néanmoins, les résultats sont discordants entre les études selon le moment de l'injection des CSM. L'amélioration clinique ne se voit qu'en cas d'injection préventive avant l'induction de l'arthrite complète. Ainsi, il a été rapporté qu'une seule injection de CSM primaires issues de moelle osseuse empêche l'apparition de l'arthrite grave, ce qui a été associé à une diminution dans le sérum des cytokines pro-inflammatoires et à une augmentation des T régulateurs (Treg) [7]. Des résultats similaires ont été obtenus à partir de CSM isolées du tissu adipeux (ADSC) qui suppriment la réponse des lymphocytes T via l'activation des cellules Treg spécifiques de l'antigène. Néanmoins, si l'injection des CSM est trop tardive, leurs effets immunosuppresseurs sont abolis par l'inflammation locale (TNF $\alpha$ ) et cette dernière en est même majorée [8]. II n'y a pas actuellement d'études cliniques chez l'homme évaluant l'effet des CSM dans la polyarthrite rhumatoïde.

\section{CSM et cellules progénitrices des chondrocytes}

II a également été proposé que les CSM puissent être utilisées comme des cellules progénitrices des chondrocytes pour la régénération du cartilage [9]. Caplan et al. ont montré que, après leur implantation dans le condyle fémoral, les CSM incluses dans une matrice collagène régénèrent en partie le cartilage lésé mais il existe une discontinuité entre les nouveaux tissus et ceux de l'hôte [10]. Les CSM autologues ont été injectées par voie intra-articulaire après l'induction d'une arthrose expérimentale. Après 26 semaines, les auteurs ont constaté une prévention des lésions chondrales mettant en évidence les propriétés paracrines des CSM [11]. Des études cliniques phase $1 / 2$ contrôlées contre placébo sont en cours par injection intra-articulaire de CSM allogéniques (produit Prochymal ${ }^{\circledast} \mathrm{d}^{\prime} 0$ siris).

On peut s'attendre à des études de faisabilité dans des indications de maladies auto-immunes rares comme les vascularites systémiques, la sclérodermie, la maladie de Gougerot-Sjögren. En effet, un bénéfice thérapeutique peut être attendu en raison de l'effet pro-angiogénique des CSM et des effets antifibrosant, anti-inflammatoire et immunosuppresseur induits par ces cellules.

\section{GVHD}

Dans la GVHD (graft versus host disease), une étude préliminaire utilisant des CSM fait état d'une réduction significative de la mortalité à 2 ans [12] $(\rightarrow)$. $(\rightarrow)$ Voir l'article de C. Ménard et $K$. Tarte, page 269 de ce numéro
Ces résultats prometteurs ont conduit à une étude plus large randomisée, contrôlée contre placebo, mais les résultats n'ont pas permis de confirmer l'efficacité des CSM comme traitement en première ligne pour traiter la GvHD corticorésistante. Le traitement a cependant entraîné une amélioration significative chez les patients ayant une atteinte hépatique ou gastro-intestinale dans le cadre de la GVHD et une amélioration cliniquement significative par rapport aux témoins dans une population pédiatrique [13].

\section{Conclusion}

La régénération de tissus endommagés dans différentes situations pathologiques et inflammatoires est un objectif majeur à atteindre dans un proche avenir et elle pourrait bénéficier des propriétés réparatrices et immunomodulatrices des CSM. La régénération complète d'un tissu est en effet un objectif difficile à atteindre et comprend l'intégration du tissu nouvellement régénéré au sein des tissus receveurs et la récupération de sa capacité fonctionnelle. Dans le cas de la thérapie cellulaire des maladies dysimmunitaires, les CSM sont les meilleurs candidats. Enfin, les données récentes montrent que le bénéfice thérapeutique repose sur les propriétés paracrines médiées par les facteurs sécrétés. Afin de progresser dans ces applications thérapeutiques, une collaboration pluridisciplinaire est indispensable afin d'optimiser la production des CSM, les doses, le suivi in vivo des cellules injectées et l'identification des mécanismes physiopathologiques impliqués. $\diamond$

\section{RÉFÉRENCES}

1. Liang J, Zhang $\mathrm{H}$, Hua B, et al. Allogenic mesenchymal stem cells transplantation in refractory systemic lupus erythematosus: a pilot clinical study. Ann Rheum Dis $2010 ; 69: 1423-9$.

2. Carrion F, Nova $E$, Ruiz C, et al. Autologous mesenchymal stem cell treatment increased $T$ regulatory cells with no effect on disease activity in two systemic lupus erythematosus patients. Lupus $2010 ; 19: 317-22$.

3. Zappia $\varepsilon$, Casazza S, Pedemonte $\varepsilon$, et al. Mesenchymal stem cells ameliorate experimental autoimmune encephalomyelitis inducing T-cell anergy. Blood 2005 ; $106: 1755-61$.

4. Karussis D, Karageorgiou C, Vaknin-Dembinsky A, et al. Safety and immunological effects of mesenchymal stem cell transplantation in patients with multiple sclerosis and amyotrophic lateral sclerosis. Arch Neurol 2010; 67 : 1187-94

5. Yamout B, Hourani R, Salti H, et al. Bone marrow mesenchymal stem cell transplantation in patients with multiple sclerosis: a pilot study. J Neuroimmunol $2010 ; 227: 185-9$.

6. Garcia-Olmo D, Herreros D, Pascual I, et al. Expanded adipose-derived stem cells for the treatment of complex perianal fistula: a phase II clinical trial. Dis Colon Rectum $2009 ; 52: 79-86$

7. Augello A, Tasso R, Negrini SM, et al. Cell therapy using allogeneic bone marrow mesenchymal stem cells prevents tissue damage in collagen-induced arthritis. Arthritis Rheum 2007 ; 56 : 1175-86.

8. Djouad F, Fritz V, Apparailly F, et al. Reversal of the immunosuppressive properties of mesenchymal stem cells by tumor necrosis factor alpha in collagen-induced arthritis. Arthritis Rheum 2005 ; 52 : 1595-1603.

9. Wakitani S, Goto T, Pineda SJ, et al. Mesenchymal cell-based repair of large, fullthickness defects of articular cartilage. J Bone Joint Surg 1994 ; 76A : 579-92

10. Caplan Al, Elyaderani M, Mochizuki Y, et al. Principles of cartilage repair and regeneration. Clin Orthopaedics Related Res 1997 ; $342: 254-69$.

11. Murphy JM, Fink DJ, Hunziker EB, Barry FP. Stem cell therapy in a caprine model of osteoarthritis. Arthritis Rheum $2003 ; 48: 3464-74$.

12. Le Blanc K, Rasmusson I, Sundberg B, et al. Treatment of severe acute graftversus-host disease with third party haploidentical mesenchymal stem cells. Lancet 2004 ; 363 : 1 439-41.

13. Le Blanc K, Frassoni F, Ball L, et al. Mesenchymal stem cells for treatment of steroid-resistant, severe, acute graft-versus-host disease: a phase II study. Lancet 2008 ; $371: 1579-86$. 
Cellules souches mésenchymateuses

et régénération du tissu osseux

Frédéric Deschaseaux

\section{Formation du tissu osseux à partir des CSM}

Le tissu osseux se forme lors de la squelettogenèse qui débute par la condensation de cellules mésenchymateuses. Puis selon le type d'os à construire, os long ou os plat, la formation osseuse progresse via deux mécanismes différents nommés ossification membranaire ou endochondrale. L'ossification membranaire se fait en une seule étape de différenciation directe des cellules condensées en ostéoblastes alors que l'ossification endochondrale se fait en deux étapes: la formation de cartilage précède la néo-ostéogenèse. Ces deux mécanismes s'appliquent aux cellules multipotentes, les CSM, qui donneront donc les cellules du cartilage (chondrocytes) et les cellules capables de synthétiser la matrice osseuse, les ostéoblastes (pour revue, voir [1]). Ces CSM sont visibles lors de la squelettogenèse en périphérie des structures condensantes (périchondrales ou ostéales). Cette capacité d'ossification endochondrale et membranaire est conservée tout au long de la vie d'un individu du stade fœtal au stade adulte, y compris chez le sujet même très âgé (bien qu'avec semble-t-il une moindre efficacité). Ainsi, il est possible d'utiliser les CSM comme cellules réparatrices dans certaines pathologies du tissu osseux comme l'ostéoporose (qu'elle soit liée à l'âge ou non), les défauts de consolidation après une fracture, les pertes importantes de substance osseuse (post-traumatique ou à la suite d'une exérèse tumorale), les maladies d'origine génétique comme l'ostéogenèse imparfaite ( 0 l ou maladie des os de verre), les ostéonécroses, etc. Le but est donc de reconstituer un tissu osseux fonctionnel assurant pleinement ses différents rôles dans le métabolisme du calcium, le support d'organes et comme niche d'un certain nombre de cellules souches et progéniteurs adultes (cellules souches hématopoïétiques, progéniteurs endothéliaux, progéniteurs vasculaires).

\section{Stratégies d'utilisation des CSM pour la reconstruction osseuse}

\section{Les atouts des CSM natives}

Pour ce faire, il existe plusieurs approches qui dépendront de l'indication clinique. La première est une approche basée sur un tri (par cytométrie de flux ou colonnes magnétiques) direct de CSM à partir d'échantillons de moelle osseuse ou de tissu adipeux. Grâce à la connaissance d'un (ou plusieurs) marqueur moléculaire spécifiquement exprimé à la surface des membranes des CSM, il est possible de concevoir des billes magnétiques recouvertes d'anticorps monoclonaux dirigés contre ce marqueur. Les CSM pourraient alors être retenues (sélection positive) sur des colonnes apposées contre un aimant (ce principe est utilisé en hématologie pour trier des cellules souches hématopoïétiques $\mathrm{CD}_{34}{ }^{+}$utilisées dans le traitement de cer- taines hémopathies). Cela permettrait d'obtenir des greffons très riches en CSM de type natif (contrairement aux CSM cultivées) qui pourraient être injectés aux patients à traiter. L'obstacle principal auquel se heurte cette démarche est le faible nombre de cellules obtenu après ces tris, ce qui peut sérieusement limiter leur utilisation en clinique humaine. En effet, on estime la fréquence des CSM à $1 / 10^{5}$ cellules mononucléées dans la moelle osseuse chez l'adulte, donc très inférieure à la fréquence des cellules hématopoïétiques $\operatorname{CD} 34^{+}$que I'on utilise pour certaines greffes en hématologie [2]. Très récemment, des CSM natives ont été isolées chez la souris et transplantées dans des souris irradiées létalement. Contrairement aux CSM cultivées, les CSM natives ont repeuplé efficacement la moelle osseuse et le tissu adipeux. De plus, dans ces expériences, elles ont reconstitué les tissus même après plusieurs transplantations en série, ce qui prouve leur capacité d'autorenouvellement [3]. Ainsi, le faible nombre de CSM natives pourrait être compensé après transplantation par leur plus forte capacité régénérative. Mais il reste que les CSM natives sont encore peu connues et des laboratoires de recherche tentent actuellement de les caractériser très précisément. II faut quand même noter que certains protocoles cliniques - notamment dans le retard de consolidation ou la pseudarthrose des os longs qui survient après une fracture - utilisent la moelle osseuse fraîche concentrée (donc débarrassée d'une bonne partie de son contenu en globules rouges). Concentrer la moelle osseuse revient à enrichir un peu un greffon en CSM natives ayant une capacité de réparation ostéogénique. Historiquement, Connolly en 1989 a montré chez l'animal une corrélation positive entre la capacité ostéogénique de la moelle osseuse et sa concentration cellulaire [4]. Healey (1990) et Connolly (1991) rapportaient des observations de consolidation de pseudarthroses induite par la seule injection de moelle osseuse autologue $[5,6]$. Cette technique a été améliorée par Hernigou en 1997, qui a centrifugé la moelle osseuse prélevée et a réinjecté seulement la portion contenant les cellules nucléées. Dans un article publié en 2005, Hernigou démontrait que la consolidation d'une pseudarthrose était fonction du nombre de cellules ostéoprogénitrices injectées (sous-entendu les CSM natives) $[7,8]$. Plus le nombre de colonies issues de CSM ou de CFU-F (colony forming unit-fibroblast) était élevé dans la moelle osseuse concentrée, plus important était le taux de consolidation des pseudarthroses. Cela démontre que les CSM natives, dont sont issues les CFU-F, peuvent être suffisamment concentrées par des techniques simples pour induire la guérison d'une pathologie de l'os. 


\section{Les atouts des CSM cultivées}

Les CSM cultivées offrent plusieurs aouts: la population est pure et il est possible d'obtenir un très grand nombre de cellules dans des conditions sécurisées de grade clinique $[9,19](\rightarrow)$. Les expériences in vitro comme in vivo chez l'animal démontrent qu'elles sont capables de régénérer le tissu osseux seules ou accompagnées de supports biocompatibles [20] $(\rightarrow)$.

Cependant, les études chez l'homme se font plus rares et une bonne partie étaient compassionnelles. Parmi les études, il faut retenir celle effectuée en plusieurs étapes par l'équipe d'Horwitz pour traiter des enfants atteints d'ostéogenèse imparfaite [10, 11]. Ces enfants ont d'abord été traités par injection de moelle osseuse allogénique puis ultérieurement par des CSM cultivées et génétiquement modifiées pour intégrer un gène rapporteur. À chaque traitement, des améliorations significatives du tableau clinique des patients ont été observées: augmentation de croissance, augmentation de la densité osseuse et diminution des fractures. Ce type de protocole a également été utilisé pour traiter un fœtus de 32 semaines atteint d'0I, les CSM allogéniques amplifiées ex vivo étant injectées in utero [12]. Cet enfant, à 2 ans, a une croissance similaire à celle d'un enfant sain de même âge.

Un élément qui doit être pris en compte est la dureté du tissu osseux. La perte, même partielle, de ce matériel doit être compensée par un apport de biomatériaux capables de servir de support aux cellules mais capables aussi de supporter certaines contraintes mécaniques. La perte de substance osseuse peut être compensée par l'ajout simultané de biomatériaux et de CSM cultivées, ou de cellules ostéoblastiques (issues de CSM dont la différenciation a été induite in vitro). Le premier essai chez l'homme a été réalisé chez trois patients ayant eu des pertes de substance osseuse à la suite de divers traumatismes. Des CSM autologues amplifiées in vitro ont été ajoutées à un biomatériau (hydroxyapatite) puis greffées, ce qui a permis aux patients une bonne reconstitution des membres atteints [13].

Cependant, il n'y a pas eu, dans cette étude, de témoins contrôles traités par le biomatériau seul, sans CSM. De façon similaire, des essais de reconstitution de mâchoire ont été tentés. Notamment, Warnke et al. ont confectionné un implant ostéorégénératif composé de CSM sur un support titane/hydroxyapatite imbibé de facteur ostéogénique recombinant humain BMP-7 (bone morphogenetic protein) [14]. Ce protocole est une résultante de la motivation des chirurgiens maxillo-faciaux à introduire ces nouvelles technologies dans leur pratique, notamment pour reconstruire la mâchoire, le tissu périodontal ou pour l'ostéoplastie de fentes palatines [15, 16].

Dans d'autres études, des CSM cultivées in vitro ont été utilisées pour combler des pertes de substances consécutives à des procédures d'allongement de membres ou de résection de tumeurs $[17,18]$. Dans l'ensemble les résultats sont encourageants mais ils restent insuffisants, notamment dans la conduite d'essais rigoureux, ce qui rend difficile les analyses à court et à long terme. Le développement de protocoles cliniques associant CSM cultivées et biomatériaux un peu partout dans le monde, notamment en Europe, nous permettra sans doute de répondre à un certains nombre de questions et surtout contribuera à améliorer notre prise en charge des pathologies du tissu osseux. $\diamond$

\section{RÉFÉRENCES}

1. Deschaseaux F, Pontikoglou C, Sensebe L. Bone regeneration: the stem/ progenitor cells point of view. J Cell Mol Med 2010 ; 14 : 103-15.

2. Pittenger MF, Mackay AM, Beck SC, et al. Multilineage potential of adult human mesenchymal stem cells. Science 1999 ; 284 : 143-7.

3. Morikawa S, Mabuchi Y, Kubota Y, et al. Prospective identification, isolation, and systemic transplantation of multipotent mesenchymal stem cells in murine bone marrow. J Exp Med 2009 ; 206 : 2483-96.

4. Connolly J, Guse R, Lippiello L, Dehne R. Development of an osteogenic bone-marrow preparation. J Bone Joint Surg Am 1989 ; 71 : 684-91.

5. Connolly JF, Guse R, Tiedeman J, Dehne R. Autologous marrow injection as a substitute for operative grafting of tibial nonunions. Clin Orthop Relat Res $1991 ; 256: 259-70$.

6. Healey JH, Zimmerman PA, McDonnell JM, Lane JM. Percutaneous bone marrow grafting of delayed union and nonunion in cancer patients. Clin Orthop Relat Res $1990 ; 256: 280-5$.

7. Hernigou P, Beaujean F. Pseudarthrosis treated by percutaneous autologous bone marrow graft. Rev Chir Orthop Reparatrice Appar Mot 1997 ; $83: 495-504$.

8. Hernigou P, Poignard A, Beaujean F, Rouard H. Percutaneous autologous bone-marrow grafting for nonunions. Influence of the number and concentration of progenitor cells. J Bone Joint Surg Am 2005; 87 : 1430-7.

9. Sensebe L, Bourin P. Mesenchymal stem cells for therapeutic purposes. Transplantation 2009 ; 87: S49-53.

10. Horwitz EM, Gordon PL, Koo WK, et al. Isolated allogeneic bone marrowderived mesenchymal cells engraft and stimulate growth in children with osteogenesis imperfecta: Implications for cell therapy of bone. Proc Natl Acad Sci USA $2002 ; 99: 8932-7$.

11. Horwitz EM, Prockop DJ, Fitzpatrick LA, et al. Transplantability and therapeutic effects of bone marrow-derived mesenchymal cells in children with osteogenesis imperfecta. Nat Med 1999 ; 5 : 309-13.

12. Le Blanc K, Gotherstrom C, Ringden 0 , et al. Fetal mesenchymal stem-cell engraftment in bone after in utero transplantation in a patient with severe osteogenesis imperfecta. Transplantation 2005 ; 79 : 1607-14.

13. Quarto R, Mastrogiacomo M, Cancedda R, et al. Repair of large bone defects with the use of autologous bone marrow stromal cells. N EnglJ Med 2001 ; $344: 385-6$.

14. Warnke PH, Springer IN, Wiltfang J, et al. Growth and transplantation of a custom vascularised bone graft in a man. Lancet $2004 ; 364: 766-70$.

15. Yamada Y, Ueda M, Hibi H, Baba S. A novel approach to periodontal tissue regeneration with mesenchymal stem cells and platelet-rich plasma using tissue engineering technology: A clinical case report. Int J Periodontics Restorative Dent 2006 ; $26: 363-9$.

16. Hibi H, Yamada Y, Ueda M, Endo Y. Alveolar cleft osteoplasty using tissueengineered osteogenic material. Int J Oral Maxillofac Surg 2006 ; $35: 551-5$.

17. Kitoh H, Kitakoji T, Tsuchiya H, et al. Transplantation of marrow-derived mesenchymal stem cells and platelet-rich plasma during distraction osteogenesis: a preliminary result of three cases. Bone $2004 ; 35: 892-8$.

18. Morishita T, Honoki $K$, Ohgushi H, et al. Tissue engineering approach to the treatment of bone tumors: three cases of cultured bone grafts derived from patients' mesenchymal stem cells. Artif Organs 2006 ; 30 : 115-8.

19. Sensebé L, Bourin P.Cellules souches mésenchymateuses : production à usage clinique et contraintes sécuritaires. Med Sci (Paris) $2011 ; 27$ : 297 302.

20. Vinatier C, Bordenave L, Guicheux J, Amédée J. Les cellules souches en ingénierie des tissus ostéoarticulaires et vasculaires. Med Sci (Paris) 2011 ; 27 : 289-96. 
Les cellules souches mésenchymateuses

de moelle osseuse et du tissu adipeux

en médecine régénératrice cardiovasculaire

Valérie Planat-Benard

\section{Potentiel cardiovasculaire des CSM}

Dans le domaine des maladies cardiovasculaires, la thérapie cellulaire se positionne comme une alternative prometteuse aux traitements actuels et elle représente en outre une thématique passionnante pour la recherche. De nombreuses études se sont concentrées sur des populations cellulaires non sélectionnées de la moelle osseuse - les cellules mononucléées médullaires ou BM-MNC - et des tissus adipeux - fraction stromale vasculaire ou SVF. Ces fractions cellulaires ont été et sont encore testées dans des protocoles cliniques de thérapie cellulaire pour le traitement de pathologies cardiovasculaires. En effet, elles représentent des sources abondantes et facilement accessibles de cellules à potentiel thérapeutique. Cependant, l'hétérogénéité de ces fractions brutes non purifiées nécessite de grandes quantités de cellules car les cellules d'intérêt y sont diluées, ce qui réduit considérablement l'efficacité thérapeutique. Les cellules souches mésenchymateuses (CSM) présentent une alternative pertinente à ces fractions cellulaires non triées. Outre les potentiels connus de différenciation des CSM dans les voies adipogénique, ostéogénique et chondroblastique, les CSM semblent également capables de différenciation en des phénotypes myocytaires et vasculaires dans des conditions de culture appropriées. Leur administration semble faciliter la réparation cardiaque comme la néovascularisation, contribuant à l'amélioration de la fonction ventriculaire. Les mécanismes sous-jacents à ces effets bénéfiques semblent majoritairement indirects via des sécrétions paracrines, et plus modestement liés à des capacités de transdifférenciation de ces cellules $[36](\rightarrow)$.

\section{Mécanismes cellulaires des CSM pour la régénération cardiaque}

\section{Différenciation des CSM en cardiomyocytes}

La première démonstration de la différenciation de CSM in vitro en cardiomyocytes contractiles a été obtenue après traitement de ces cellules par la 5 -azacytidine, un agent hypométhylant $[1,2]$. En effet, ce traitement induit dans les cellules une ultrastructure de cardiomyocyte, de type myotube, et celles-ci expriment de multiples caractéristiques des cellules cardiaques: expression de facteurs de transcription (Nkx2.5, GATA4, T\&F-1 et MEF-2c), de protéines spécifiques (peptides natriurétiques, myosines, troponines, connexines), de récepteurs adrénergiques et muscariniques capables de moduler la rythmicité des contractions, développement de potentiels d'action caractéristiques. L'obtention d'une différenciation cardiomyocytaire en l'absence de 5-azacytidine à laquelle on substitue divers facteurs de croissance (vascular endothelial growth factor, VEGF ; transforming growth factor beta, TGF- $\beta$ ) ou agents inducteurs (acide butyrique, acide rétinoïque) ne permet qu'une différenciation incomplète et un phénotype immature, suggérant que les facteurs de différenciation requis et les mécanismes moléculaires ne sont pas tous identifiés.

Une autre stratégie permettant de diriger le devenir des cellules immatures est de les placer dans un système de coculture avec les cellules différenciées d'intérêt. Les CSM, mises en présence de cardiomyocytes néonatals, adoptent ce phénotype, ce qui a conforté I'hypothèse que des événements de transdifférenciation peuvent survenir in vitro [7-9]; il faut toutefois prendre en considération des mécanismes additionnels, comme la fusion cellulaire et la reprogrammation qui peuvent également contribuer à l'émergence de cardiomyocytes à partir des CSM. Dans ces systèmes de coculture il a été démontré que la sécrétion de facteurs mais aussi le contact entre les différents types de cellules sont requis $[11,12]$. Toutefois, le degré de maturité du phénotype cardiaque des cellules obtenues dans ces protocoles de coculture est là aussi débattu. On pense que les CSM se différencieraient plutôt en des cellules biphénotypiques intermédiaires qui exprimeraient des marqueurs spécifiques cardiaques tout en conservant un phénotype stromal, mimant ainsi le cardiomyocyte mais sans acquérir toutefois l'ensemble de ses fonctions [14].

Une situation considérée comme très favorable pour orienter le devenir des cellules vers un phénotype d'intérêt consiste à administrer les cellules in vivo dans un contexte cellulaire de régénération, par exemple dans un tissu lésé. Ainsi, les CSM ont été injectées dans des modèles d'ischémie cardiaque mais les données sont contradictoires: certaines sont en faveur de l'acquisition de marqueurs cardiaques par les CSM, alors que d'autres ne montrent aucun signe de cardiogenèse par transdifférenciation in vivo, en particulier à partir de CSM de tissu adipeux. Compte tenu des incertitudes concernant le devenir des cellules injectées et la faible quantité de cellules implantées, la réelle capacité de différenciation des CSM en nouveaux cardiomyocytes in vivo reste donc très discutée et des arguments en faveur de sa réalité restent encore à apporter.

\section{Rôle trophique indirect}

\section{des CSM dans la réparation cardiaque}

L'ensemble des données recueillies dans les modèles animaux d'infarctus du myocarde mais aussi dans des modèles non ischémiques de cardiopathie 
dilatée, de cryolésion, d'arythmie ou de défaut des valves cardiaques s'accordent toutefois à démontrer le bénéfice fonctionnel et tissulaire associé à l'administration de CSM. Dans les cœurs qui ont reçu des CSM, la cicatrice fibreuse est de plus petite taille, la viabilité des myocytes préservée et la néo-angiogenèse augmentée [18-24]. Les modèles animaux ont permis d'identifier quelles propriétés des CSM contribuaient à améliorer significativement la fonction ventriculaire. Ainsi, le rôle trophique des CSM fait intervenir les remarquables capacités sécrétoires de ces cellules: facteurs de croissance capables éventuellement de mobiliser des progéniteurs cardiaques endogènes, chémokines (SDFl ou CCL12) permettant de recruter des progéniteurs circulants, facteurs anti-apoptotiques cardioprotecteurs (hepatocyte growth factor [HGF], secreted frizzled-related protein 2 [Sfrp2], Aktl, insulin growth factor 1 [IGFl]) qui améliorent la survie des myocytes et limitent dans le temps le développement de l'insuffisance cardiaque. Les CSM sécrètent aussi des facteurs pro-angiogéniques (VEGF, fibroblast growth factor [FGF2], angiopoïétine-1) qui participent à la néovascularisation du myocarde, ce qui se traduit par une augmentation de la densité vasculaire dans les cœurs traités par des CSM. Les CSM produisent des métalloprotéases (MMP-9, MMP-3) actives sur le remodelage matriciel qui limite la fibrose. Enfin les CSM jouent un rôle-clé dans la résolution de l'immuno-inflammation par leurs propriétés immunomodulatrices [27, 37] $(\rightarrow)$.

En particulier, les CSM diminuent l'expression et la sécrétion de cytokines inflammatoires (tumor necrosis factor alpha [TNF- $\alpha$ ], $\mathrm{IL}-6$ ), inhibent la formation de collagène de types I et III ainsi que l'expression des protéines MMP-1 et TIMP-1 (tissue inhibitor of metalloproteinases). Une augmentation de la germination du nerf cardiaque a été démontrée dans un modèle porcin d'infarctus du myocarde, suggérant également la sécrétion de facteurs neurotrophiques par les CSM administrées [28].

\section{Mécanismes cellulaires des CSM pour la régénération vasculaire}

Ils découlent des modèles d'ischémie in vivo (cardiaque, membre postérieur, cérébrale ou encore cutanée) qu'un effet majeur des CSM est de promouvoir la néo-angiogenèse et d'améliorer ainsi la perfusion tissulaire. La différenciation en cellules de type endothélial a été obtenue in vitro à partir de CSM de moelle osseuse [29] et de tissu adipeux $[30,31]$. Elle se manifeste en culture par la constitution de réseaux structurés d'alignements cellulaires caractéristiques et l'acquisition de marqueurs spécifiques, tels que les récepteurs VEGF-R1 (FLT-1) et VEGF-R2 (KDR ou Flk-1), le facteur von Willebrand, ou le CD31. Injectées in vivo, les CSM sont associées à une augmentation de la circulation cutanée mesurée par doppler laser, du score angiographique et de la densité capillaire. Des arguments en faveur d'une transdifférenciation en cellules endothéliales ont été observés in vivo [30-32], suggérant que les CSM peuvent directement contribuer à la formation de néo-vaisseaux, même si ces événements restent encore une fois modestes et ne peuvent pas rendre compte à eux seuls de la néo-vascularisation des tissus ischémiques. Ces effets sont favorisés par la secrétion paracrine, par les CSM, de facteurs pro-angiogéniques $[33,34]$. Un point remarquable est que la production de ces facteurs, du VEGF en particulier, peut être sensiblement améliorée par un préconditionnement hypoxique (généralement une exposition de 24 heures à $1 \%$ d' $\mathrm{O}_{2}$ ) ou génétiquement. L'administration comparative de CSM de moelle osseuse ou de tissu adipeux dans un modèle porcin d'infarctus du myocarde aigu [21], dans un modèle d'infarctus du myocarde chronique chez le rat [24] ou dans l'ischémie de patte [35] amène à la conclusion que les cellules issues de tissu adipeux sont plus efficaces pour stimuler la néo-angiogenèse que les cellules issues de moelle osseuse.

La preuve d'une contribution significative des CSM à la réparation cardiaque et vasculaire a été apportée dans de multiples modèles précliniques. Cependant leur capacité à générer des cellules fonctionnelles capables de remplacer les cardiomyocytes endommagés ou détruits et de produire de nouveaux vaisseaux semble limitée. Leurs effets bénéfiques sont plutôt associés à un rôle «stromal », un rôle de soutien qui tend à rétablir une homéostasie tissulaire via des sécrétions paracrines et des mécanismes régulateurs, afin de préserver la fonction contractile ventriculaire et une bonne perfusion tissulaire tout en modulant la réponse inflammatoire délétère. Les CSM représentent donc des candidats intéressants pour les thérapies cellulaires des pathologies cardiovasculaires. L'utilisation des CSM de moelle osseuse est actuellement en cours dans quelques essais cliniques de thérapie cellulaire. Les CSM de tissu adipeux sont actuellement testées dans deux essais de faisabilité et d'innocuité pour le traitement de l'ischémie critique chronique des membres inférieurs en France et en Espagne. De nombreux aspects devront toutefois être optimisés dans un contexte clinique, comme les modes d'administration et la survie des cellules après transplantation. La recherche fondamentale doit surtout apporter des réponses quant au mode d'action de ces populations de cellules, à la sécurité de leur isolement et de leur production ex vivo [38] $(\rightarrow)$, sur leur innocuité et devenir à long terme et enfin sur leurs propriétés immunomodulatrices et leur potentielle utilisation allogénique. $\diamond$

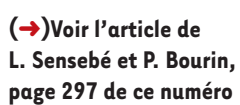




\section{RÉFÉRENCES}

1. Makino S, Fukuda K, Miyoshi S, et al. Cardiomyocytes can be generated from marrow stromal cells in vitro. J Clin Invest $1999 ; 103: 697-705$.

2. Rangappa $\mathrm{S}$, Fen $\mathrm{C}$, Lee $\varepsilon \mathrm{H}$, et al. Transformation of adult mesenchymal stem cells isolated from the fatty tissue into cardiomyocytes. Ann Thorac Surg $2003 ; 75: 775-9$.

7. Li H, Yu B, Zhang $Y$, et al. Jaggedl protein enhances the differentiation of mesenchymal stem cells into cardiomyocytes. Biochem Biophys Res Commun 2006 ; 341 : 320-5.

8. Wang T, Xu Z, Jiang W, et al. Cell-to-cell contact induces mesenchymal stem cell to differentiate into cardiomyocyte and smooth muscle cell. Int J Cardiol $2006 ; 109: 74-81$.

9. Arminan A, Gandia C, Bartual M, et al. Cardiac differentiation is driven by NKX2.5 and GATA4 nuclear translocation in tissue-specific mesenchymal stem cells. Stem Cells Dev 2009; $18: 907-18$.

11. Wang $T, X u Z$, Jiang $W$, et al. Cell-to-cell contact induces mesenchymal stem cell to differentiate into cardiomyocyte and smooth muscle cell. Int J Cardiol 2006 ; 109 : 74-81.

12. Zhu Y, Liu T, Song K, et al. ADSCs differentiated into cardiomyocytes in cardiac microenvironment. Mol Cell Biochem 2009 ; 324 : 117-29.

14. Rose RA, Jiang $\mathrm{H}$, Wang $\mathrm{X}$, et al. Bone marrow-derived mesenchymal stromal cells express cardiacspecific markers, retain the stromal phenotype and do not become functional cardiomyocytes in vitro. Stem Cells $2008 ; 26: 2884-92$

18. Grauss RW, Winter EM, van Tuyn J, et al. Mesenchymal stem cells from ischemic heart disease patients improve left ventricular function after acute myocardial infarction. Am J Physiol Heart Circ Physiol 2007 ; 293 : H2438-47.

19. Berry MF, Engler AJ, Woo YJ, et al. Mesenchymal stem cell injection after myocardial infarction improves myocardial compliance. Am J Physiol Heart Circ Physiol 2006 ; 290 : H2196-2203.

20. Jaquet $K$, Krause KT, Denschel J, et al. Reduction of myocardial scar size after implantation of mesenchymal stem cells in rats: what is the mechanism? Stem Cells Dev 2005; 14 : 299-309.

21. Valina C, Pinkernell K, Song $\mathrm{YH}$, et al. Intracoronary administration of autologous adipose tissuederived stem cells improves left ventricular function, perfusion, and remodelling after acute myocardial infarction. Eur HeartJ $2007 ; 28: 2667-77$.

22. Cai L, Johnstone BH, Cook TG, et al. IFATS collection: human adipose tissue-derived stem cells induce angiogenesis and nerve sprouting following myocardial infarction, in conjunction with potent preservation of cardiac function. Stem Cells $2009 ; 27: 230-7$.

23. Noiseux N, Gnecchi M, Lopez-Ilasaca M, et al. Mesenchymal stem cells overexpressing akt dramatically repair infarcted myocardium and improve cardiac function despite infrequent cellular fusion or differentiation. Mol Ther $2006 ; 14: 840-50$.

24. Mazo M, Planat-Benard V, Abizanda G, et al. Transplantation of adipose derived stromal cells is associated with functional improvement in a rat model of chronic myocardial infarction. EurJ Heart Fail $2008 ; 10: 454-62$.

27. Caplan Al, Dennis JE. Mesenchymal stem cells as trophic mediators. J Cell Biochem 2006 ; $98: 1076-84$

28. Pak HN, Dayyum M, Kim DT, et al. Mesenchymal stem cell injection induces cardiac nerve sprouting and increased tenascin expression in a Swine model of myocardial infarction. J Cardiovasc Electrophysiol 2003; $14: 841-8$.

29. Oswald J, Boxberger S, Jørgensen B, et al. Mesenchymal stem cells can be differentiated into endothelial cells in vitro. Stem Cells $2004 ; 22: 377-84$.

30. Planat-Benard V, Silvestre JS, Cousin B, et al. Plasticity of human adipose lineage cells toward endothelial cells: physiological and therapeutic perspectives. Circulation 2004 ; $109: 656-63$

31. Miranville A, Heeschen C, Sengenes C, et al. Improvement of postnatal neovascularization by human adipose tissue-derived stem cells. Circulation $2004 ; 110: 349-55$.

32. Quevedo HC, Hatzistergos KE, Oskouei BN, et al. Allogeneic mesenchymal stem cells restore cardiac function in chronic ischemic cardiomyopathy via trilineage differentiating capacity. Proc Natl Acad Sci USA 2009; 106 : 14022-7.

33. Feygin J, Mansoor A, Eckman $\mathrm{P}$, et al. Functional and bioenergetic modulations in the infarct border zone following autologous mesenchymal stem cell transplantation. Am J Physiol Heart Circ Physiol 2007 ; 293 : H1772-80.

34. Rehman J, Traktuev D, Li J, et al. Secretion of angiogenic and antiapoptotic factors by human adipose stromal cells. Circulation $2004 ; 109: 1292-8$.

35. Kim Y, Kim H, Cho H, et al. Direct comparison of human mesenchymal stem cells derived from adipose tissues and bone marrow in mediating neovascularization in response to vascular ischemia. Cell Physiol Biochem $2007 ; 20: 867-76$.

36. Charbord P, Casteilla L. La biologie des cellules souches mésenchymateuses d'origine humaine. Med Sci (Paris) $2011 ; 27: 261-8$.

37. Ménard C, Tarte K. Immunosuppression et cellules souches mésenchymateuses : mieux comprendre une propriété thérapeutique majeure. Med Sci (Paris) 2011 ; 27 : 269-74.

38. Sensebé $L$, Bourin P.Cellules souches mésenchymateuses : production à usage clinique et contraintes sécuritaires. Med Sci (Paris) 2011 ; 27 : 297-302.

\section{Les cellules souches mésenchymateuses et la médecine régénérative en ophtamologie Éric Gabison}

Dans le domaine de l'ophtalmologie, les cellules souches mésenchymateuses représentent une nouvelle voie de recherche avec des applications possibles dans les pathologies de la cornée mais également de la rétine et du nerf optique.

\section{CSM et maladies de la cornée}

La cornée, première lentille transparente localisée à la surface de l'œil, a été l'un des tissus précurseurs dans le domaine de la thérapie cellulaire. Les opacités cornéennes associées aux insuffisances en cellules souches épithéliales limbiques (ICSL) 3 ont fait l'objet d'études chez l'homme dès la fin des années 1990 [1, 2]. Si les formes strictement unilatérales d'ICSL sont généralement traitées par une autogreffe de limbe provenant de l'œil sain, les formes bilatérales ont longtemps été des impasses thérapeutiques. S'il persiste des cellules prélevables sur au moins l'un des deux yeux, les cellules épithéliales cornéennes peuvent être amplifiées au laboratoire, puis les feuillets épithéliaux cultivés greffés, avec des résultats satisfaisants. Dans le cas d'atteintes bilatérales, des cellules épithéliales de muqueuse buccale ont été utilisées, amplifiées puis greffées sur les cornées lésées, ce qui a permis de restaurer une transparence cornéenne compatible avec l'autonomie des patients traités [3]. Cependant, ces techniques ne sont pas réalisées en routine et le pronostic de ce type de thérapie cellulaire se heurte à de nombreux échecs, notamment en cas de pathologies inflammatoires chroniques de la surface oculaire. Les CSM sont particulièrement étudiées in vitro et in vivo pour leurs propriétés anti-inflammatoires et antifibrotiques ainsi que pour leur rôle de soutien de la croissance des cellules souches épithéliales $(\rightarrow)$ Voir l'article de C. Ménard et K. Tarte et, page 269 de ce numéro $[4,27](\rightarrow)$.

Des cellules souches mésenchymateuses ayant de nombreuses homologies avec les CSM issues de la moelle osseuse ont d'ailleurs été identifiées au niveau de la périphérie cornéenne où elles pourraient participer au microenvironnement - à la «niche »-des cellules sou-

${ }^{3}$ Le limbe cornéen est à la jonction de la conjonctive et de la cornée périphérique. C'est là que se trouveraient les cellules souches épithéliales. 


\section{RÉFÉRENCES}

1. Makino S, Fukuda K, Miyoshi S, et al. Cardiomyocytes can be generated from marrow stromal cells in vitro. J Clin Invest $1999 ; 103: 697-705$.

2. Rangappa $\mathrm{S}$, Fen $\mathrm{C}$, Lee $\varepsilon \mathrm{H}$, et al. Transformation of adult mesenchymal stem cells isolated from the fatty tissue into cardiomyocytes. Ann Thorac Surg $2003 ; 75: 775-9$.

7. Li H, Yu B, Zhang $Y$, et al. Jaggedl protein enhances the differentiation of mesenchymal stem cells into cardiomyocytes. Biochem Biophys Res Commun 2006 ; 341 : 320-5.

8. Wang T, Xu Z, Jiang W, et al. Cell-to-cell contact induces mesenchymal stem cell to differentiate into cardiomyocyte and smooth muscle cell. Int J Cardiol $2006 ; 109: 74-81$.

9. Arminan A, Gandia C, Bartual M, et al. Cardiac differentiation is driven by NKX2.5 and GATA4 nuclear translocation in tissue-specific mesenchymal stem cells. Stem Cells Dev 2009; $18: 907-18$.

11. Wang $T, X u Z$, Jiang $W$, et al. Cell-to-cell contact induces mesenchymal stem cell to differentiate into cardiomyocyte and smooth muscle cell. Int J Cardiol 2006 ; 109 : 74-81.

12. Zhu Y, Liu T, Song K, et al. ADSCs differentiated into cardiomyocytes in cardiac microenvironment. Mol Cell Biochem 2009 ; 324 : 117-29.

14. Rose RA, Jiang $\mathrm{H}$, Wang $\mathrm{X}$, et al. Bone marrow-derived mesenchymal stromal cells express cardiacspecific markers, retain the stromal phenotype and do not become functional cardiomyocytes in vitro. Stem Cells $2008 ; 26: 2884-92$

18. Grauss RW, Winter EM, van Tuyn J, et al. Mesenchymal stem cells from ischemic heart disease patients improve left ventricular function after acute myocardial infarction. Am J Physiol Heart Circ Physiol 2007 ; 293 : H2438-47.

19. Berry MF, Engler AJ, Woo YJ, et al. Mesenchymal stem cell injection after myocardial infarction improves myocardial compliance. Am J Physiol Heart Circ Physiol 2006 ; 290 : H2196-2203.

20. Jaquet $K$, Krause KT, Denschel J, et al. Reduction of myocardial scar size after implantation of mesenchymal stem cells in rats: what is the mechanism? Stem Cells Dev 2005; 14 : 299-309.

21. Valina C, Pinkernell K, Song $\mathrm{YH}$, et al. Intracoronary administration of autologous adipose tissuederived stem cells improves left ventricular function, perfusion, and remodelling after acute myocardial infarction. Eur HeartJ $2007 ; 28: 2667-77$.

22. Cai L, Johnstone BH, Cook TG, et al. IFATS collection: human adipose tissue-derived stem cells induce angiogenesis and nerve sprouting following myocardial infarction, in conjunction with potent preservation of cardiac function. Stem Cells $2009 ; 27: 230-7$.

23. Noiseux N, Gnecchi M, Lopez-Ilasaca M, et al. Mesenchymal stem cells overexpressing akt dramatically repair infarcted myocardium and improve cardiac function despite infrequent cellular fusion or differentiation. Mol Ther $2006 ; 14: 840-50$.

24. Mazo M, Planat-Benard V, Abizanda G, et al. Transplantation of adipose derived stromal cells is associated with functional improvement in a rat model of chronic myocardial infarction. EurJ Heart Fail $2008 ; 10: 454-62$.

27. Caplan Al, Dennis JE. Mesenchymal stem cells as trophic mediators. J Cell Biochem 2006 ; $98: 1076-84$

28. Pak HN, Dayyum M, Kim DT, et al. Mesenchymal stem cell injection induces cardiac nerve sprouting and increased tenascin expression in a Swine model of myocardial infarction. J Cardiovasc Electrophysiol 2003; $14: 841-8$.

29. Oswald J, Boxberger S, Jørgensen B, et al. Mesenchymal stem cells can be differentiated into endothelial cells in vitro. Stem Cells $2004 ; 22: 377-84$.

30. Planat-Benard V, Silvestre JS, Cousin B, et al. Plasticity of human adipose lineage cells toward endothelial cells: physiological and therapeutic perspectives. Circulation 2004 ; $109: 656-63$

31. Miranville A, Heeschen C, Sengenes C, et al. Improvement of postnatal neovascularization by human adipose tissue-derived stem cells. Circulation $2004 ; 110: 349-55$.

32. Quevedo HC, Hatzistergos KE, Oskouei BN, et al. Allogeneic mesenchymal stem cells restore cardiac function in chronic ischemic cardiomyopathy via trilineage differentiating capacity. Proc Natl Acad Sci USA 2009; 106 : 14022-7.

33. Feygin J, Mansoor A, Eckman $\mathrm{P}$, et al. Functional and bioenergetic modulations in the infarct border zone following autologous mesenchymal stem cell transplantation. Am J Physiol Heart Circ Physiol 2007 ; 293 : H1772-80.

34. Rehman J, Traktuev D, Li J, et al. Secretion of angiogenic and antiapoptotic factors by human adipose stromal cells. Circulation $2004 ; 109: 1292-8$.

35. Kim Y, Kim H, Cho H, et al. Direct comparison of human mesenchymal stem cells derived from adipose tissues and bone marrow in mediating neovascularization in response to vascular ischemia. Cell Physiol Biochem $2007 ; 20: 867-76$.

36. Charbord P, Casteilla L. La biologie des cellules souches mésenchymateuses d'origine humaine. Med Sci (Paris) $2011 ; 27: 261-8$.

37. Ménard C, Tarte K. Immunosuppression et cellules souches mésenchymateuses : mieux comprendre une propriété thérapeutique majeure. Med Sci (Paris) 2011 ; 27 : 269-74.

38. Sensebé $L$, Bourin P.Cellules souches mésenchymateuses : production à usage clinique et contraintes sécuritaires. Med Sci (Paris) 2011 ; 27 : 297-302.

\section{Les cellules souches mésenchymateuses et la médecine régénérative en ophtamologie Éric Gabison}

Dans le domaine de l'ophtalmologie, les cellules souches mésenchymateuses représentent une nouvelle voie de recherche avec des applications possibles dans les pathologies de la cornée mais également de la rétine et du nerf optique.

\section{CSM et maladies de la cornée}

La cornée, première lentille transparente localisée à la surface de l'œil, a été l'un des tissus précurseurs dans le domaine de la thérapie cellulaire. Les opacités cornéennes associées aux insuffisances en cellules souches épithéliales limbiques (ICSL) 3 ont fait l'objet d'études chez l'homme dès la fin des années 1990 [1, 2]. Si les formes strictement unilatérales d'ICSL sont généralement traitées par une autogreffe de limbe provenant de l'œil sain, les formes bilatérales ont longtemps été des impasses thérapeutiques. S'il persiste des cellules prélevables sur au moins l'un des deux yeux, les cellules épithéliales cornéennes peuvent être amplifiées au laboratoire, puis les feuillets épithéliaux cultivés greffés, avec des résultats satisfaisants. Dans le cas d'atteintes bilatérales, des cellules épithéliales de muqueuse buccale ont été utilisées, amplifiées puis greffées sur les cornées lésées, ce qui a permis de restaurer une transparence cornéenne compatible avec l'autonomie des patients traités [3]. Cependant, ces techniques ne sont pas réalisées en routine et le pronostic de ce type de thérapie cellulaire se heurte à de nombreux échecs, notamment en cas de pathologies inflammatoires chroniques de la surface oculaire. Les CSM sont particulièrement étudiées in vitro et in vivo pour leurs propriétés anti-inflammatoires et antifibrotiques ainsi que pour leur rôle de soutien de la croissance des cellules souches épithéliales $(\rightarrow)$ Voir l'article de C. Ménard et K. Tarte et, page 269 de ce numéro $[4,27](\rightarrow)$.

Des cellules souches mésenchymateuses ayant de nombreuses homologies avec les CSM issues de la moelle osseuse ont d'ailleurs été identifiées au niveau de la périphérie cornéenne où elles pourraient participer au microenvironnement - à la «niche »-des cellules sou-

${ }^{3}$ Le limbe cornéen est à la jonction de la conjonctive et de la cornée périphérique. C'est là que se trouveraient les cellules souches épithéliales. 
ches limbiques et avoir une action trophique sur ces cellules. Des études expérimentales chez l'animal décrivent l'effet bénéfique des CSM sur la cicatrisation cornéenne après application de milieu conditionné de CSM ou même après injection de CSM dans le tissu lésé [5-9]. En dehors de ce rôle de modulation de la cicatrisation cornéenne et de protection du microenvironnement des cellules souches épithéliales cornéennes résidentes, certaines études rapportent la transdifférenciation des CSM $(\rightarrow)$ [28] en cellules épithéliales in vitro ou même in vivo lorsqu'elles sont greffées sur une surface oculaire blessée. De telles propriétés ont été observées dans des modèles de brûlures chimiques chez le rat et le lapin. Les CSM pourraient ainsi représenter une nouvelle source cellulaire dans le traitement des ICSL sévè- $(\rightarrow)$ Voir l'article de P. Charbord et L. Casteilla, page 261 de ce numéro res $[10-12]$.

Les opacités cornéennes du stroma (derme) cornéen pourraient également être traitées par injection de CSM. L'organisation semi-cristalline de la matrice extracellulaire cornéenne et les propriétés de faible diffraction de la lumière des kératocytes (fibroblastes qui synthétisent cette matrice) concourent à la transparence cornéenne. Si l'injection de fibroblastes cutanés dans le stroma cornéen est à l'origine d'une inflammation et d'une perte de transparence cornéenne, des études ont démontré la survie et la différenciation en kératocytes de CSM médullaires injectées au niveau du stroma cornéen et la préservation de la transparence cornéenne. De plus, dans des modèles murins d'opacités cornéennes héréditaires, l'injection de CSM humaines a permis la restauration de la transparence des cornées traitées [13-16]. Ces cellules humaines, injectées dans le stroma de souris déficientes en lumican (un protéoglycane de la matrice extracellulaire), étaient capables d'y produire la forme humaine de ce protéoglycane et de rétablir l'organisation matricielle stricte nécessaire à la transmission de la lumière et à la transparence cornéenne.

\section{Cellules souches mésenchymateuses et maladies rétiniennes}

Les propriétés thérapeutiques des CSM ont également été évaluées au cours des pathologies rétiniennes. Chez le rat, dans un modèle de rétinopathie pigmentaire héréditaire, l'injection de CSM dans l'espace sous rétinien ou par voie intraveineuse a permis de ralentir l'évolution de la dégénérescence rétinienne [17-21]. Dans le même contexte, une autre approche élégante consiste à réaliser des injections intraoculaires de CSM génétiquement modifiées pour produire des facteurs de neuroprotection [22]. Dans ces différents modèles expérimentaux, l'effet bénéfique rapporté n'était pas constamment associé à la survie au long cours des CSM. En effet, si in vitro ces cellules peuvent exprimer certains marqueurs de cellules de l'épithélium pigmentaire ou même de photorécepteurs, la différenciation des CSM en cellules rétiniennes observée dans certaines études n'est néanmoins pas nécessaire à leur effet thérapeutique in vivo, soulignant ainsi l'importance de leur rôle trophique.

Cet effet neuroprotecteur des CSM a également été démontré dans des pathologies traumatiques ou dégénératives (glaucome chronique à angle ouvert) du nerf optique, dans des modèles murins. Dans ces études, les CSM étaient d'origine humaine ou autologue, génétiquement modifiées ou non pour la production de facteurs neurotrophiques [23, 24].

L'intérêt croissant pour les CSM en ophtalmologie repose sur leurs propriétés immunomodulatrices, anti-inflammatoires, trophiques et sur leur plasticité. Ces propriétés sont particulièrement adaptées au traitement des pathologies oculaires de la cornée au nerf optique. $\diamond$

\section{SUMMARY}

Mesenchymal stem cells: a therapeutic update

Mesenchymal stem cells/multipotent marrow stromal cells (MSC) have the ability to participate in the reconstruction of tissues both directly by providing repair cells (essentially those originating from mesoderm) and indirectly by modulating inflammatory and immune responses. This wide range of properties makes these cells very appealing to treat various pathological conditions. They have been first used in 1995 as supportive cells to facilitate hematopoietic stem cells engraftment, and then to minimize the deleterious consequences of graft versus host disease by their immunosuppressive function. Their robust osteogenic differentiation capacity has also been evaluated in numerous preclinical settings of healing/repair but more rarely in human clinical trials. During the past 10-15 years, the potential benefit of their paracrine actions has been tested in various situations such as to facilitate repair after cutaneous defects after burns or lower consequences of ischemic strokes. The purpose of this series of short texts is not to give an exhaustive panorama, but to discuss some well-identified indications in four different fields: auto-immune diseases, bone repair, vascular regeneration and eye lesions such as corneal and retinal defects. $\diamond$

\section{CONFLIT D'INTÉRÊTS}

Les auteurs déclarent n 'avoir aucun conflit d'intérêts concernant les données publiées dans cet article.

\section{RÉFÉRENCES}

1. Pellegrini G, Traverso CE, Franzi AT, et al. Long-term restoration of damaged corneal surfaces with autologous cultivated corneal epithelium. Lancet $1997 ; 349: 990-3$.

2. Tsai RJ, Li LM, Chen JK. Reconstruction of damaged corneas by transplantation of autologous limbal epithelial cells. N Engl J Med 2000 ; 343: 86-93.

3. Nishida K, Yamato M, Hayashida Y, et al. Corneal reconstruction with tissueengineered cell sheets composed of autologous oral mucosal epithelium. $N$ Engl J Med 2004 ; 351 : 1187-96.

4. Zhang $X$, Sun $H$, Li X, Yuan X, et al. Utilization of human limbal mesenchymal cells as feeder layers for human limbal stem cells cultured on amniotic membrane. J Tissue Eng Regen Med 2010 ; 4 : 38-44.

5. Reinshagen H, Auw-Haedrich C, Sorg RV, et al. Corneal surface reconstruction using adult mesenchymal stem cells in experimental limbal stem cell deficiency in rabbits. Acta Ophthalmol 2011 (sous presse). 


\section{RÉFÉRENCES}

6. Watson SL, Marcal H, Sarris M, et al. The effect of mesenchymal stem cell conditioned media on corneal stromal fibroblast wound healing activities. Br J Ophthalmol $2010 ; 94: 1067-73$.

7. Ye J, Yao K, Kim JC. Mesenchymal stem cell transplantation in a rabbit corneal alkali burn model: engraftment and involvement in wound healing. Eye (Lond) $2006 ; 20: 482-90$.

8. Gabison $\varepsilon \varepsilon$, Zhang $X$, Huet $\varepsilon$, et al. Therapeutic potential of bone marrow mesenchymal stem cells in corneal chemical burns: An in vitro and in vivo study. Invest Ophthalmol Vis Sci 2009; $50: 3067$.

9. Choong PF, Mok PL, Cheong SK, et al. Mesenchymal stromal cell-like characteristics of corneal keratocytes. Cytotherapy $2007 ; 9: 252-8$.

10. Funderburgh ML, Du Y, Mann MM, et al. PAX6 expression identifies progenitor cells for corneal keratocytes. FASEBJ $2005 ; 19: 1371-3$.

11. Jiang TS, Cai L, Ji WY, et al. Reconstruction of the corneal epithelium with induced marrow mesenchymal stem cells in rats. Mol Vis 2010 ; 16 : 1304-16.

12. Gu S, Xing C, Han J, et al. Differentiation of rabbit bone marrow mesenchymal stem cells into corneal epithelial cells in vivo and ex vivo. Mol Vis $2009 ; 15: 99-107$

13. Ma Y, Xu Y, Xiao Z, et al. Reconstruction of chemically burned rat corneal surface by bone marrowderived human mesenchymal stem cells. Stem Cells $2006 ; 24: 315-21$.

14. Du Y, Carlson EC, Funderburgh ML, et al. Stem cell therapy restores transparency to defective murine corneas. Stem Cells $2009 ; 27: 1635-42$.

15. Arnalich-Montiel F, Pastor S, Blazquez-Martinez A, et al. Adipose-derived stem cells are a source for cell therapy of the corneal stroma. Stem Cells $2008 ; 26: 570-9$.

16. Du Y, Funderburgh ML, Mann MM, et al. Multipotent stem cells in human corneal stroma. Stem Cells $2005 ; 23: 1266-75$

17. Liu H, Zhang J, Liu CY, et al. Cell therapy of congenital corneal diseases with umbilical mesenchymal stem cells: lumican null mice. PLoS One 2010 ; 5 : el0707.

18. Wang $S$, Lu B, Girman $S$, et al. Non-invasive stem cell therapy in a rat model for retinal degeneration and vascular pathology. PLoS One. $2010 ; 5$ : e9200.

19. Lu B, Wang $S$, Girman $S$, et al. Human adult bone marrow-derived somatic cells rescue vision in a rodent model of retinal degeneration. Exp Eye Res $2010 ; 91: 449-55$

20. Li N, Li XR, Yuan JQ. Effects of bone-marrow mesenchymal stem cells transplanted into vitreous cavity of rat injured by ischemia/reperfusion. Graefes Arch Clin Exp Ophthalmol 2009 $247: 503-14$
21. Inoue $Y$, Iriyama A, Ueno $S$, et al. Subretinal transplantation of bone marrow mesenchymal stem cells delays retinal degeneration in the RCS rat model of retinal degeneration. Exp Eye Res $2007 ; 85: 234-41$.

22. Arnhold $S$, Absenger $Y$, Klein $H$, et al. Transplantation of bone marrowderived mesenchymal stem cells rescue photoreceptor cells in the dystrophic retina of the rhodopsin knockout mouse. Graefes Arch Clin Exp Ophthalmol $2007 ; 245: 414-22$

23. Harper MM, Adamson L, Blits B, et al. Brain-derived neurotrophic factor released from engineered mesenchymal stem cells attenuates glutamateand hydrogen peroxide-mediated death of staurosporine-differentiated RGC-5 cells. Exp Eye Res $2009 ; 89$ : 538-48.

24. Johnson TV, Bull ND, Hunt DP, et al. Neuroprotective effects of intravitreal mesenchymal stem cell transplantation in experimental glaucoma. Invest Ophthalmol Vis Sci $2010 ; 51: 2051-9$.

25. Zwart I, Hill AJ, Al-Allaf F, et al. Umbilical cord blood mesenchymal stromal cells are neuroprotective and promote regeneration in a rat optic tract model. Exp Neurol 2009 ; 216 : 439-48.

26. Kicic $A$, Shen $W$, Wilson $A S$, et al. Differentiation of marrow stromal cells into photoreceptors in the rat eye. J Neurosci $2003 ; 23$ : 7742-9.

27. Ménard C, Tarte K. Immunosuppression et cellules souches mésenchymateuses : mieux comprendre une propriété thérapeutique majeure. Med Sci (Paris) $2011 ; 27$ : 269-74.

28. Charbord P, Casteilla L. La biologie des cellules souches mésenchymateuses d'origine humaine. Med Sci (Paris) $2011 ; 27: 261-8$.

\section{Le plus pratique des lexiques médicaux trilingues}

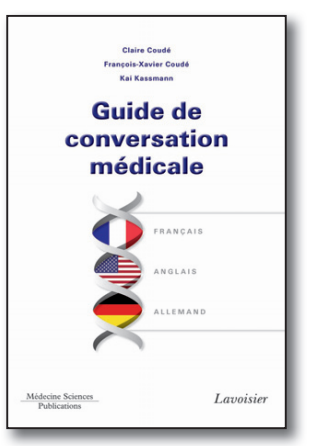

15/04/2011

Prix Public TTC : $59 €$

416 pages - broché

ISBN : 978-2-257-20421-9

\section{Guide de conversation médicale Français-Anglais-Allemand} Claire COUDÉ, François-Xavier COUDÉ, Kaï KASSMANN

Ce Guide de conversation médicale trilingue est l'outil nécessaire à tout médecin, de langue maternelle française, anglaise ou allemande, souhaitant séjourner dans un milieu hospitalier francophone, anglophone ou germanophone. Le livre s'articule autour de trois parties :

- Conversation médicale concerne le vocabulaire utilisé lors de l'examen du patient et de la discussion entre médecins, classé par spécialités. Les expressions courantes et familières ont également été retenues.

- Lexique médical réunit toute la terminologie indispensable à linterrogatoire du patient, à la rédaction du compte rendu ou à la demande d'examens de laboratoire, ainsi que les abréviations médicales habituellement rencontrées.

- Annexes regroupe des renseignements pratiques médicaux (médicaments classés par DCI avec leurs noms commerciaux français, anglais et allemands, planches d'anatomie trilingues...) ou de la vie courante.

41 sections composent ainsi le Guide de conversation médicale. Chaque section est présentée en trois colonnes - une par langue - et repérée par un numéro, permettant des renvois de l'une à l'autre.

En vente chez votre libraire spécialisé, par correspondance ou sur notre site www.medecine.lavoisier.fr

Bon de commande à retourner complété à : LAVOISIER SAS - 14, rue de Provigny - 94236 CACHAN Cedex

Guide de conversation médicale: $59 €$ TTC

(+ $7 €$ de participation aux frais de port par exemplaire) soit $66 €$

Frais de port offerts* à partir de $60 €$ si paiement joint à la commande
( France métropolitaine, Suisse, UE. Autres, nous consulter par mail : info@Lavoisier.fr)

Carte bancaire $n^{\circ}$

Quantité

Les 3 derniers chiffres situés au dos de votre carte bancaire

Fonction / spécialité :

Code postal :

$\ldots \ldots \ldots$

.. . Ville : . . .

Adresse :

Ces renseignements pourront figurer sur un fichier informatique. Conformément à la loi Informatique \& Libertés du 6 janvier 1978, vous bénéficiez d'un droit d'accès et de rectification aux données vous concernant.

Date d'expiration :

Nom / Prénom :

\section{Je joins mon règlement à la commande :}

Montant total de :

$€$

Chèque bancaire ou postal payable en France à l'ordre de : LAVOISIER SAS (Une facture acquittée sera jointe au colis)

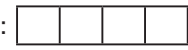

C.Jorgensen,

. Deschaseaux,

V. Planat-Benard

et $\varepsilon$. Gabison 